\title{
Research article \\ HIV Myelopathy- A cross-sectional study of constellation of bone marrow findings in HIV/AIDS
}

\author{
Hemalatha Kuppan ${ }^{1}$, Vanishri Ashok ${ }^{2}$, Sridevi Hanaganahalli Basavaiah ${ }^{3}$, Urmila Niranjan Khadilkar², Nirupama \\ Murali $^{4}$ \\ ${ }^{1}$ Junior Resident (DNB), Department of Pathology, Apollo Hospital, Chennai, Tamil Nadu, India \\ ${ }^{2}$ Consultant Pathologist, Hematology Section, Central Laboratory, Kasturba Medical College Hospitals, Ambedkar Circle, \\ Mangalore, Karnataka, India \\ ${ }^{3}$ Additional Professor, Department of Pathology, Kasturba Medical College, Mangalore, Manipal Academy of Higher \\ Education, Manipal, Karnataka, India - 576104 \\ ${ }^{4}$ Professor and Head, Department of Pathology, Dr. Chandramma Dayananda Sagar Institute of Medical Education and \\ Research, Devarakaggalahalli, Harohalli, Kanakapura Road, Ramanagara Dt., Karnataka - 562112
}

(Received: August 2021

Revised: September 2021

Accepted: November 2021)

Corresponding author: Sridevi Hanaganahalli Basavaiah. sridevi.somesh@manipal.edu

\begin{abstract}
Introduction and Aim: Haematological manifestations in HIV disease is common and can happen at any phase during the disease course. Anemia and thrombocytopenia are the most frequent hematologic abnormalities and are associated with high morbidity and mortality. The objective of current study was to observe and analyse various spectrum of bone marrow changes and haematological abnormalities in HIV/AIDS and to correlate findings with CD4 count.
\end{abstract}

Material and Methods: A total of 44 patients over a period of 5 years were included. Clinical findings, hematological profile, bone-marrow aspirate, biopsy findings and CD4 count of these patients were documented. The association between absolute lymphocyte count (ALC) and CD4 count were further established.

Results: The most common clinical indication for bone-marrow aspiration and trephine biopsy was pancytopenia (47.3\%), pyrexia of unknown origin (15.1\%), and unresolving hepatosplenomegaly (13.6\%). Anemia (72.7\%) was commonest haematological abnormality. Bone marrow aspirate was normocellular in majority of patients. Marrow findings were correlated with CD4 count and were found to be statistically significant. Tri-lineage dysplasia was observed in $9.1 \%$ of patients, and megakaryocytic dysplasia being the commonest $(61.4 \%)$. Histiocytic aggregates (27.3\%) were noted among which $6.8 \%$ showed acid fast bacilli in Ziehl-Neelsen stain. Fungal stains revealed histoplasmosis in $4.5 \%$ patients.

Conclusion: There was a strong negative association between presence of anemia and dysplasia and CD4 count. When CD4 was $<200 / \mu \mathrm{L}$ and ALC $<1000 / \mathrm{mm}^{3}$, presence of anemia and dysplasia affecting various cell lines were commonly observed; therefore, can be used as indicators to assess the severity of the disease.

Keywords: Dysplasia; anaemia; HIV; thrombocytopenia; bone marrow.

\section{INTRODUCTION}

$\mathrm{H}$ uman immunodeficiency virus (HIV) infection is a true pandemic affecting individuals all over the world (1). The first case of acquired immunodeficiency syndrome (AIDS) was identified in United States in 1981, and in 1983, HIV was isolated from a case of lymphadenopathy (2). In the year 2019, there were 38 million people living with HIV (PLHIV) globally with 1.7 million new cases and 6,90,000 AIDS-related deaths documented by the end of the year (3). India, being a developing country with considerably large population and low socioeconomic prominence, has the third largest HIV-positive population. India reported 23.48 lakh cases of PLHIV in 2019 and there were 69.22 thousand new cases with adult HIV prevalence of $0.22 \%$ between $15-49$ years. National AIDS-related deaths documented in 2019 was 58.96 thousand (4).
HIV/AIDS is a multisystem disease with wide range of clinical and pathological changes, primarily involving a sub-group of T-lymphocytes (CD4+ helper $\mathrm{T}$ subset). According to Centre for disease control and prevention (CDC) definition, the diagnosis of AIDS is considered when CD4 lymphocytes count goes below 200 cells $/ \mu \mathrm{L}$ or patients develop either any illness documented under category $\mathrm{C}$ or specific AIDS associated diseases $(3,4)$.

Hematological abnormalities are common throughout the course of HIV infection and can manifest as anemia, leucopenia or thrombocytopenia. These changes can be due to direct viral effect or can be secondary to opportunistic infections, malignancies or treatment (5). Constellation of bone marrow findings that are noted in HIV/AIDS is called as HIV myelopathy (6). Several cytokines secreted through the course of HIV infection are potent inhibitors of hematopoiesis (1). Recently, it has been established 
that HIV can infect three subclasses of hematopoietic precursor cells which are multipotent hematopoietic progenitor cells, mast cell progenitor cells, and monocytic precursor cells (7).

The effect of bone marrow involvement in HIV leading to anemia and thrombocytopenia increases the risk of disease related death. Hematological workup plays a pivotal role in recognizing these abnormalities at early stages, to initiate necessary treatment and thus avoiding the associated co-morbidities. Correlation of absolute lymphocyte and CD4 counts with hematological and bone marrow changes has been barely performed in the past and can throw light into disease pathogenesis. We intended to analyze the hematological abnormalities and bone marrow changes associated with HIV disease; and correlation of marrow changes with absolute lymphocyte count (ALC) and CD4 count.

\section{MATERIALS AND METHODS}

\section{Study plan and study population}

We conducted a retrospective observational analysis in the department of Pathology of a tertiary care center of Southern India over a period of 5 years. Patients who were HIV-positive diagnosed by enzyme-linked immunosorbent assay (ELISA) method, as per NACO guidelines, and, had underwent bone marrow examination formed the study population. The bone marrow aspiration and trephine biopsy slides were retrieved from the archives and were reviewed. The HIV cases without any hematological abnormalities, and/or without bone marrow evaluation were excluded from the study. Baseline clinical details were obtained from the medical records. Institutional Ethics Committee approval was obtained prior to the study.

\section{Laboratory methods}

Blood cell counts performed by Beckman Coulter® LH780 Cellular Analysis System (LH780; Miami, Florida, USA), a completely automated hematology analyzer, were obtained. The parameters such as hemoglobin, white blood cell count, differential WBC count, platelet count, RBC indices- mean corpuscular volume, mean corpuscular haemoglobin concentration, mean corpuscular haemoglobin, hematocrit and absolute lymphocyte count (ALC) were recorded. Peripheral smear smears were stained with Leishman stain and findings were recorded. Bone marrow aspirates (BMA) stained with Leishman stain and bone marrow biopsy (BMB) slides stained with hematoxylin-eosin (H\&E) stain were analysed and findings were documented. Histochemical stains such as Ziehl Neelson stain, Gomori Methamine Silver stain, Periodic Acid Schiff Stain, Mucicarmine stain and Prussian blue stain were performed on BMB wherever necessary. Bone marrows were reviewed for cellularity, myeloid to erythroid (M:E) ratio, trilineage hematopoiesis for their number and maturation, plasmacytosis, eosinophilia, individual lineage hyperplasia and/or dysplasia, abnormal lymphoid cells, serous atrophy, marrow fibrosis, naked nuclei of megakaryocytes, histiocytic aggregates, granulomas, fungal spores, and infections such as tuberculosis and histoplasmosis. Absolute lymphocyte count was obtained from Beckman Coulter and CD4 counts were measured using flow cytometry Beckman Coulter FC500 analyzer and were correlated.

\section{Ethical clearance}

The study has obtained approval from the Institutional Ethics Committee, Kasturba Medical College, Mangalore (Reg. No. ECR/541/Inst/KA/2014/RR17), with a reference number- IEC KMC MLR 0816/158. The study did not involve any direct patient interrogation and only retrospective review of the slides were performed. Informed consent from the participants was not taken and was waived by the IRB due to the retrospective nature of the study.

\section{Statistical analysis}

Statistical analysis of the data obtained was performed using SPSS software version 22. Baseline analysis was expressed as mean or as percentage frequencies. Data comparison was performed using Chi square test and student-t test. We considered a probability value of $<0.05$ to be statistically significant.

\section{RESULTS}

\section{Baseline demographic characteristics}

During the study duration, a total of 44 HIV patients fulfilled the inclusion criteria who had undergone both hematological and bone marrow evaluation. Mean age of HIV infected cases was 40.9 years (age range: 12 70 years) with peak occurrence between $31-50$ years (79.4\%). There were 33 males and 11 females with a $\mathrm{M}: \mathrm{F}$ ratio of $3: 1$. In these patients, bone marrow examination was performed as a part of workup for pyrexia of unknown origin (PUO), unresolving hepatosplenomegaly or as a part of evaluation of refractory anemia, leucopenia, neutropenia, lymphopenia or thrombocytopenia. The most common complaint in these patients were cough $(86.4 \%)$, fever $(84.1 \%)$ and breathlessness (72.7\%). Hepatosplenomegaly was seen in $13.6 \%$ cases.

\section{Associated infections and neoplasms}

Clinically, among the 44 HIV cases, 25 cases were associated with tuberculosis $(56.8 \%)$ and were receiving anti-tubercular therapy. CMV retinitis (11.4\%), oral candidiasis (15.9\%), Non Hodgkin Lymphoma (4.5\%), toxoplasmosis (4.5\%) and MAC infection (4.5\%) and Hodgkin lymphoma (2.3\%) were the other associations. However, bone marrow evaluation of these cases revealed granulomas, infections (tuberculosis, histoplasmosis) and malignancies (acute leukemia) in $31.8 \%$ of cases. 


\section{Complete blood count and peripheral smear findings}

Anemia $(72.7 \%)$ was the most common abnormality followed by thrombocytopenia (50\%) and leucopenia (36.4\%). Pancytopenia was observed in $47.7 \%$ and bicytopenia in $25 \%$ cases. Monocytopenia was observed in $15.9 \%$ patients. Peripheral smear revealed microcytic hypochromic red cell morphology in $27.3 \%$ of cases followed by normocytic normochromic (27.3\%), dimorphic (11.36\%) and macrocytic (6.8\%) RBC morphology.

\section{Absolute lymphocyte and CD4 counts}

Among the 44 cases of HIV positive cases, CD4 count was $<200$ cells $/ \mu \mathrm{L}$ in $75 \%$ cases and $25 \%$ cases having $>200$ cells $/ \mathrm{mm}^{3}$. The ALC was compared with CD4 count and was found statistically significant. An ALC of $<1,000$ cells $/ \mathrm{mm}^{3}$ could predict a CD4 count to be lesser than 200 cells $/ \mathrm{mm}^{3}$ and on the other end, an ALC of $>2,000$ cells $/ \mathrm{mm}^{3}$ could predict the CD4 count to be $>200$ cells $/ \mathrm{mm}^{3}$. Fig. 1 depicts the distribution of CD4 counts by ALC.

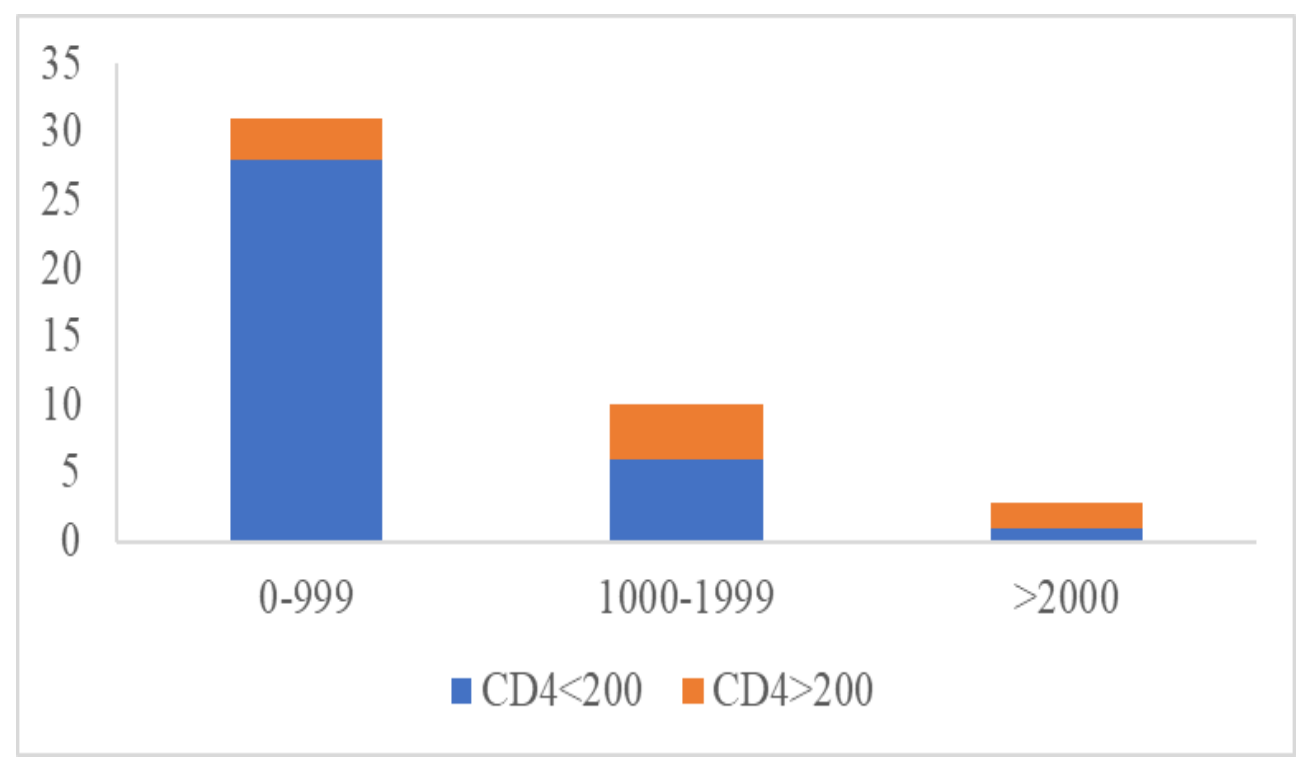

Fig. 1: Comparison of CD4 and ALC

\section{Bone marrow findings}

Bone marrow evaluation revealed various morphological changes in HIV patients (Table 1, Table 2 and Fig.2). In erythroid dysplasia, binucleation, multinucleation, cytoplasmic vacuolization, micronormoblasts and megaloblasts were noted. In myeloid series, dysplastic features such as nuclear dysmorphism, giant metamyelocytes and cytoplasmic vacuolization were observed. In megakaryocytes, hypolobulation, hyperlobulation, and clustering were noted with few cases showing naked nuclei of megakaryocytes. Table 2 shows the correlation between CD4 count with various cytopenias, cellularity and dysplasia observed in these patients. There was statistical significance between CD4 count with marrow cellularity and dysplasia. Lower CD4 count was associated with disturbed marrow cellularity and presence of either erythroid, granulocytic or megakaryocytic dysplasia, megakaryocytic being the commonest.

Table 1: Correlation of various cytopenias, marrow cellularity, dysplasia with CD4 count

\begin{tabular}{|c|c|c|c|}
\hline Findings & $\begin{array}{c}\text { Cases with } \\
\text { CD4<200 (in \%) }\end{array}$ & $\begin{array}{c}\text { Cases with } \\
\text { CD4> 200 (in \%) }\end{array}$ & Total (in \%) \\
\hline \multicolumn{4}{|c|}{ Complete blood count } \\
\hline Monocytopenia & 11.4 & 4.5 & 15.9 \\
\hline Bicytopenia & 18.2 & 6.8 & 25 \\
\hline Pancytopenia & 40.9 & 6.8 & 47.7 \\
\hline \multicolumn{4}{|c|}{ Bone marrow findings } \\
\hline Cellularity & $13.6^{*}$ & $2.3^{*}$ & $15.9^{*}$ \\
Hypercellular & 27.3 & 6.8 & 34.1 \\
Hypocellular & 25 & 20.5 & $45.5^{*}$ \\
Normocellular & 34.1 & 4.5 & 38.6 \\
\hline Dysplasia & 13.6 & 2.3 & 15.9 \\
Unilineage & 9.1 & None & 9.1 \\
Bilineage & $25^{*}$ & 6.8 & 31.8 \\
Trilineage & $18.2^{*}$ & 6.8 & 25 \\
Erythroid & $50^{*}$ & 11.4 & 61.4 \\
Myeloid & \multicolumn{3}{|c|}{} \\
Megakaryocytic &
\end{tabular}


$* \mathrm{p}$ value $<0.05$

Table 2: Various BM findings in HIV positive patients

\begin{tabular}{|c|c|c|}
\hline BM findings & No. of cases & Percentage of cases (\%) \\
\hline Plasmacytosis & 11 & 25 \\
\hline Histiocytic aggregates & 12 & 27.3 \\
\hline Reactive marrow & 5 & 11.4 \\
\hline Eosinophilia & 2 & 4.5 \\
\hline Naked nuclei of megakaryocytes & 4 & 9.1 \\
\hline Erythroid hyperplasia & 6 & 13.6 \\
\hline Myeloid hyperplasia & 2 & 4.5 \\
\hline Megakaryocytic hyperplasia & 1 & 2.3 \\
\hline Serous atrophy & 1 & 2.3 \\
\hline Granulomas & 8 & 18.2 \\
\hline Tuberculosis & 3 & 6.8 \\
\hline Histoplasmosis & 2 & 4.5 \\
\hline Leukemia & 1 & 2.3 \\
\hline
\end{tabular}

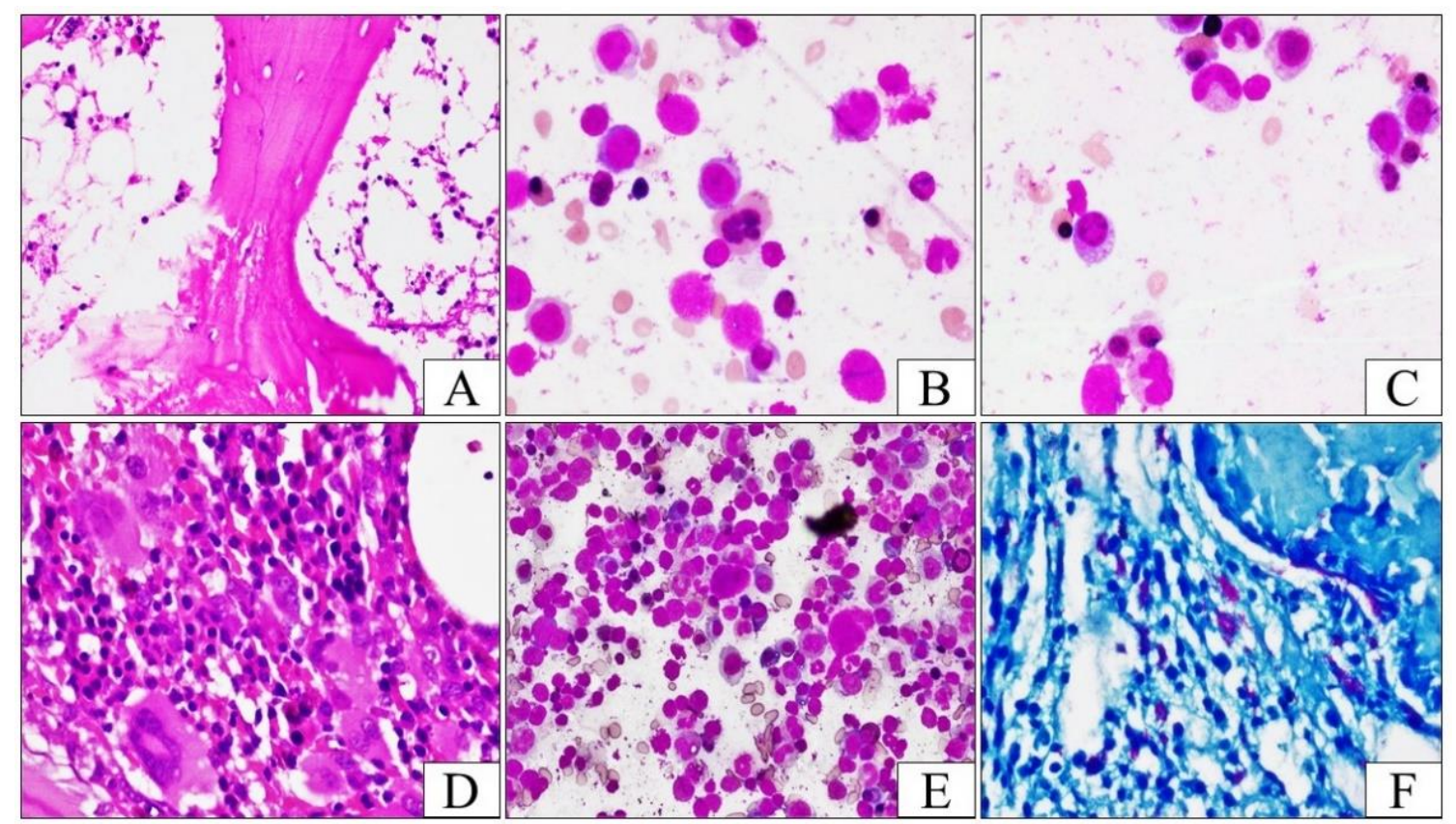

Fig. 2: Bone marrow findings. 2A: BMB with hypocellular marrow(H\&E;100x); 2B: BMA with dysplastic multinucleated erythroblast.(Leishman;200x); 2C: BMA with giant band forms, dysplastic hypogranular granulocyte, dysplastic late erythroblast with nuclear blebs(Leishman;200x); 2D: BMB with hypercellular marrow with dysplastic micro and macromegakaryocytes in clusters.(H\&E;200x); 2E: BMA with bare nuclei of megakaryocytes and plasma cells.(Leishman;100x); 2F: Ill formed granulomas with AFB positive bacilli in clusters in a HIV case with tuberculosis.(ZN;1000x)

\section{DISCUSSION}

Hematological manifestations are common during the course of HIV infection. In bone marrow, HIV infects the marrow mesenchymal stem cells, various committed progenitor cells, stromal elements, T cells and macrophages (8). The virus infected hematopoietic progenitor cells are killed by the activated $\mathrm{T}$ cells via apoptosis through Fas-ligand/Fasreceptor pathway and this is evidenced by the presence of increased Fas-ligand levels in AIDS patients (1).

Dyshematopoiesis in bone marrow (BM) is associated with peripheral blood findings such as anemia, granulocytopenia and thrombocytopenia. Although BM abnormalities are more consistently seen in HIV patients with advanced disease who are on antiretroviral therapy (ART) or associated with neoplasms and opportunistic infections; they can also occur in patients without ART, infections or malignancy. These changes occur at an early stage due to direct effect of abnormal cytokines produced by HIV infection and their impact on BM cells (9). The present study did not show existence of any infections or malignancies as a cause of hematological abnormality in $68.2 \%$ of cases.

The CD4 lymphocyte depletion is directly proportional to the plasma level of HIV viral RNA. The era of highly active ART (HAART) and its capacity of suppressing viral replication has reduced the virulence and disease progression in HIV patients. HAART has helped in increasing CD4 lymphocyte count and reducing the opportunistic infections thereby prolonging the patients' survival. However 
complete immune restitution is not possible (7). We observed $75 \%$ of cases with CD4 count $<200$ cells $/ \mathrm{mm}^{3}$. In such cases, BM evaluation forms a fundamental part of comprehensive workup in HIV patients particularly in cases of PUO, refractory anemia, pancytopenia or unexplained cytopenia, or after the initiation of ART to look for progression of developing opportunistic infections and/or neoplasms (10).

In the present study, $44 \mathrm{BMA}$ and BMB samples were examined for various parameters and $\mathrm{BM}$ examination played an important role by diagnosing the presence of opportunistic infections, neoplasia, and in evaluation of peripheral pancytopenia thereby aiding specific therapy. Tripathi et al., have reported cytopenia and PUO as the common indications for bone marrow biopsy procedure (2). In the present study unexplained or persistent cytopenia was the commonest indication. We observed patients who presented with pancytopenia had BM findings such as dysplasia in $57.1 \%$, granulomata (28.6\%), megaloblastic anemia (14.3\%), histoplasmosis (9.5\%), and acute leukemia (4.8\%). These results were consistent with findings of Santos et al., (11). In the present study, bone marrow examination in patients who presented with hepatosplenomegaly revealed NHL infiltration, tuberculosis and myelodysplasia. Among the infections causing PUO, tuberculosis was the commonest with other concomitant infections such as histoplasmosis and these findings were in concordance with earlier studies $(5,12)$.

In the present study, most of the HIV patients were drivers and coolies by occupation (54.5\%). Previous studies have found that the commonest mode of transmission of HIV in males is by commercial sex worker $(78 \%)$ and in females is from spouses $(96.6 \%)$ $(2,12)$. The anemia encountered in HIV patients is predominantly normocytic normochromic followed by microcytic hypochromic in various studies $(2,5,12)$. Anemia and neutropenia are the best clinical indicators apart from CD4 in evaluating the immune status of HIV patients (13).

Dysplastic features in the marrow ranged from 30$70 \%$ in various studies $(2,14,15)$. Tripathi et al., have reported myelodysplasia in $32.43 \%$ of AIDS patients, predominantly in granulocytic precursors $(27.03 \%)$ followed by erythroid (4.05\%) (2). In a study of 64 AIDS patients, Sharad et al., found granulocytic dysplasia in $14.06 \%$ patients, erythroid dysplasia in $12.5 \%$ patients and no megakaryocytic dysplasia was recorded (14). In the present study, dysplastic features were found in $45 \%$ patients, predominantly in megakaryocytic series followed by erythroid and granulocytic series, which is in concordance with Rudresh et al., (15). Arindham et al., found tri-lineage dysplasia in $6.52 \%$ of HIV patients as compared to $9.09 \%$ in our study (12). A statistically reliable relationship was obtained between ALC and CD4 count. In the present study, when the ALC was $<1,000$ cells $/ \mathrm{mm}^{3}$, the CD4 count was invariably $<200$ cells $/ \mathrm{mm}^{3}$, and similarly, an ALC of $>2,000$ cells $/ \mathrm{mm}^{3}$ could predict a CD4 count of $>200$ cells $/ \mathrm{mm}^{3}$ which was in concordance with Agarwal et al., (16).

Our study was limited by its retrospective nature and lack of some clinical and laboratory data such as dietary history, history of any other drugs or comorbidities, iron studies, vitamin B12 and folate levels in the serum, etc. that would have provided more light into the haematological abnormalities seen in these HIV patients.

\section{CONCLUSION}

In conclusion, the present retrospective analysis highlighted common morphological changes and haematological abnormalities in the BM of HIV patients and their correlation with CD4 count. We observed that the incidence of pancytopenia correlated with disease progression and CD4 count along with BM dysplasia. Hematopoietic dysplasias were commonly observed, even in the absence of opportunistic infections, neoplasms, or chemotherapeutic treatment, clearly suggesting that the HIV-infection is associated with dysfunctional hematopoiesis that can be secondary to direct effect of the virus and viral proteins. Additionally, the present study also revealed significant correlation of ALC with CD4 cell counts and therefore ALC can be used as an interpreter of CD4 count to assess the stage of the disease. These findings prove that hematological abnormalities are common in HIV disease and a systematic workup at early stages is necessary for investigating the cause and timely intervention for the long-time survival of people living with HIV.

\section{CONFLICT OF INTEREST}

The authors of this article declare no conflicts of interest that includes specific financial interests, relationships, and/or affiliations to the subject matters or materials included.

\section{REFERENCES}

1. Noy, A., Gulick, R. M. Acquired immunodeficiency syndrome. In: Greer JP, Arber DA, Glader B, List AF, (eds). Wintrobe's Clinical hematology. $13^{\text {th }}$ edition, Philadelphia, Wolters Kluwer, 2014: p1358-1364.

2. Tripathi, A. K., Misra, R., Kalra, P., Gupta, N., Ahmad, R Bone marrow abnormalities in HIV disease. J Assoc Physicians India 2005; 53: 705-710.

3. WHO Annual report (last update on November 30 2020). Available from: https://www.who.int/news-room/factsheets/detail/hiv-aids. [Last accessed on 2021 May 24].

4. NACO Annual report 2019-2020 (last update on July 21 2020).

5. Parintha, S. S., Kulkarni, M. H. Haematological changes in HIV infection with correlation to CD4 count. Australas Med J. 2012; 5: 157-162.

6. O’Malley, D. P., Orazi, A. Bone Marrow Abnormalities associated with Non-neoplastic Conditions. In: Hsi ED, (eds). Hematopathology, Philadelphia, Churchill Livingstone Elsevier. 2007: 173-198. 
7. McNamara, L. A., Collins, K. L. Hematopoietic stem/precursor cells as HIV reservoirs. Current Opinion in HIV and AIDS. 2011; 6: 43-48.

8. Khandekar, M. M., Deshmukh, S. D., Holla, V. V., Kakrani, A. L., Sangale, S. A., Habbu, A. A., et al., Profile of bone marrow examination in HIV/AIDS patients to detect opportunistic infections, especially tuberculosis. Indian $\mathrm{J}$ Pathol Microbiol 2005; 48: 7-12.

9. Tsukamoto, T. Hematopoietic Stem/Progenitor Cells and the Pathogenesis of HIV/AIDS. Front Cell Infect Microbiol. 2020 Feb 21; 10: 60.

10. Kotwal, C. J., Singh, M. V. A study of haematological and bone marrow changes in symptomatic patients with human immune deficiency virus infection with special mention of functional iron deficiency, anemia of critically ill and haemophagocytic lymphohistiocytosis. Med J Armed Forces 2013; 69: 319-325.

11. Santos, E. S., Raez, L. E., Eckardt, P., DeCesare, T., Whitcomb, C. C., Byrne, Jr., G. E. The utility of a bone marrow biopsy in diagnosing the source of fever of unknown origin in patients with AIDS. J Acquir Immune Defic Syndr 2004; 37: 1599-1603.

12. Pande, A., Bhattacharyya, M., Pain, S., Samanta, A. Study of bone marrow changes in antiretroviral naïve human immunodeficiency virus infected anemic patients. Indian $\mathbf{J}$ Pathol Microbiol 2011; 54: 542-546.

13. Attili, S. V. S., Singh, V. P., Rai, M., Varma, D. V., Gulati, A. K., Sundar, S. Hematological profile of HIV patients in relation to immune status - a hospital-based cohort from Varanasi, North India. Turk J Hematol. 2008; 25: 13-19.

14. Dhurve, S. A., Dhurve, S. A. Bone Marrow Abnormalities in HIV Disease. Mediterr J Hematol Infect Dis. 2013; 5910: e2013033.

15. Rudresh, K., Mysorekar, T.M.A.V.V., Modepalli, N., Ahuja, A. Bone marrow study in patients with Human Immune Deficiency Virus and Acquired Immune Deficiency Syndrome. Brunei Int Med J. 2011; 7: 148-156.

16. Agrawal, P. B., Rane, S. R., Jadhav, M. V. Absolute Lymphocyte Count as a Surrogate Marker of CD4 Count in Monitoring HIV Infected Individuals: A Prospective Study. J Clin Diagn Res. 2016 May; 10(5): EC17-19. 